\section{Lateral relocation of auxin efflux regulator PIN3 mediates tropism in Arabidopsis}

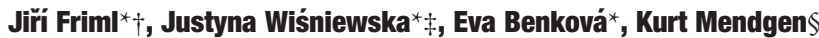
\& Klaus Palme ${ }^{\star} \|$

${ }^{*}$ Max-Delbrück-Laboratorium in der Max-Planck-Gesellschaft, 50829 Köln, Germany

$\dagger$ Zentrum für Molekularbiologie der Pflanzen, Universität Tübingen, 72076 Tübingen, Germany

$\ddagger$ Department of Biotechnology, Institute of General and Molecular Biology, 87-100 Torun, Poland

\$Lehrstuhl Phytopathologie, Universität Konstanz, 78457 Konstanz, Germany || Institut für Biologie II, Universität Freiburg, 79104 Freiberg, Germany

Long-standing models propose that plant growth responses to light or gravity are mediated by asymmetric distribution of the phytohormone auxin ${ }^{1-3}$. Physiological studies implicated a specific transport system that relocates auxin laterally, thereby effecting differential growth ${ }^{4}$; however, neither the molecular components of this system nor the cellular mechanism of auxin redistribution on light or gravity perception have been identified. Here, we show that auxin accumulates asymmetrically during differential growth in an efflux-dependent manner. Mutations in the Arabidopsis gene PIN3, a regulator of auxin efflux, alter differential growth. PIN3 is expressed in gravity-sensing tissues, with PIN3 protein accumulating predominantly at the lateral cell surface. PIN3 localizes to the plasma membrane and to vesicles that cycle in an actin-dependent manner. In the root columella, PIN3 is positioned symmetrically at the plasma membrane but rapidly relocalizes laterally on gravity stimulation. Our data indicate that PIN3 is a component of the lateral auxin transport system regulating tropic growth. In addition, actin-dependent relocalization of PIN3 in response to gravity provides a mechanism for redirecting auxin flux to trigger asymmetric growth.

Plants orientate their growth with respect to the direction of light (phototropism) or gravity (gravitropism) ${ }^{1}$. As early as 1926 a widely accepted model for plant tropisms, the Cholodny-Went hypothesis, was presented ${ }^{2}$. It proposes differential distribution of the plant hormone auxin in lateral direction on gravity or light stimulation. Subsequently, different auxin levels elicit differential growth rates, which ultimately lead to bending of the shoot or $\operatorname{root}^{3}$. Visualization of asymmetrically distributed auxin response in gravistimulated tobacco stems ${ }^{5}$ and Arabidopsis roots ${ }^{6}$ experimentally supported this hypothesis. Polar auxin transport represent a plausible means of lateral auxin distribution, as its chemical inhibition affects differential growth responses such as tropisms and apical hook formation $^{7,8}$. Physiologically characterized components of polar auxin transport are cellular efflux carriers, whose polar localization within cells is thought to determine the direction of auxin flux ${ }^{9}$. The recently identified PIN genes of Arabidopsis appear to encode essential components of these carriers ${ }^{7}$. A role of PIN2 in regulation of basipetal auxin transport and gravitropism in root ${ }^{6,10,11}$ as well as a role of PIN1 in basipetal auxin transport in the stem have been reported $^{12}$; however, so far the molecular basis of shoot tropic responses remains elusive. Lateral auxin transport with a specific, laterally localized auxin efflux carrier was proposed ${ }^{4}$ to explain the exchange of auxin between vasculature, where the main basipetal auxin stream occurs ${ }^{13}$, and peripheral tissues controlling elongation ${ }^{14}$. Nevertheless the lack of any molecular data supporting this concept still leaves the existence of such a system in question.

We analysed the relationship between auxin efflux, auxin redistribution and differential growth responses in Arabidopsis hypocotyls. Growth responses were examined and auxin levels indirectly visualized using the synthetic DR5::GUS auxin reporter ${ }^{15}$, whose activity correlates with direct auxin measurements ${ }^{16,17}$. Seedlings grown under standard conditions displayed normal phototropic and gravitropic curvature accompanied by an asymmetric DR5::GUS expression suggesting elevated auxin levels on the more elongated side of the hypocotyl (Fig. 1a, c). In contrast, seedlings that did not undergo light or gravity stimulation showed lower and uniform expression of the DR5::GUS reporter (data not shown). Seedlings subjected to light and gravity stimulation with simultaneous auxin efflux inhibition failed to show any differential DR5::GUS expression as well as tropic curvature (Fig. 1b, d). Differential DR5::GUS expression was also detected during the process of apical hook formation (Fig. 1e), and seedlings grown on auxin efflux inhibitors failed to show asymmetric auxin distribution and apical hook formation (data not shown). These results show that asymmetric growth of the shoot correlates with auxin efflux-dependent asymmetric auxin distribution, and implicate the existence of auxin efflux components involved in asymmetric growth responses.

The auxin efflux carrier candidates are PIN proteins, although their function in the regulation of differential growth has not been reported $^{7,8}$. We screened for new members of the PIN gene family and analysed corresponding knockout mutants for differential growth defects. One of the genes, PIN3, was isolated from genomic and complementary DNA libraries with probes derived from the conserved region of PIN1. The deduced PIN3 protein shares $67 \%$

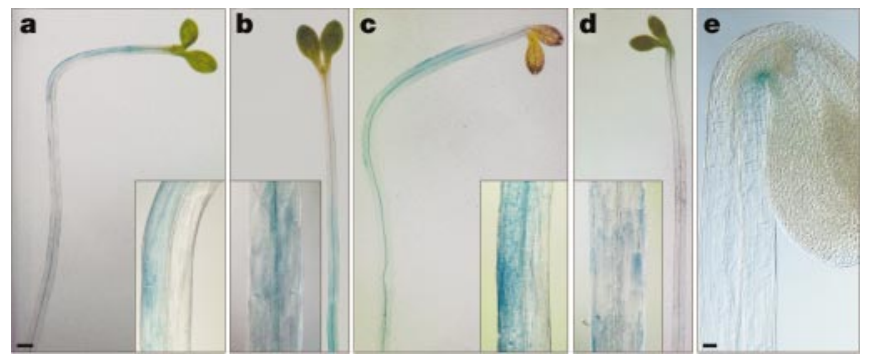

Figure 1 Auxin response during differential hypocotyl growth. $\mathbf{a}-\mathbf{d}$, Expression of the DR5:: GUS reporter in hypocotyl of untreated (a, c) or auxin efflux inhibitor (NPA)-treated (b, d) wild-type seedlings upon stimulation by light $(\mathbf{a}, \mathbf{b})$ or gravity $(\mathbf{c}, \mathbf{d})$. Insets show details of DR5::GUS expression. Scale bars, $400 \mu \mathrm{m}$. e, Asymmetric expression of DR5::GUS reporter in the apical hook of an etiolated seedling. Scale bar, $50 \mu \mathrm{m}$. 
identity to PIN1, displays the characteristic three-domain topology with about 10 transmembrane domains characteristic of all PIN proteins $^{7,8}$, and shows similarity to bacterial transporters and detoxification carriers, suggesting the same biochemical function for PIN proteins in auxin efflux ${ }^{7,8}$. By a reverse genetic strategy ${ }^{18}$ two mutant alleles designated as pin 3-1 and pin3-2 were identified (Fig. 2a). From the progeny of pin3-2 a stable, footprint mutant allele exhibiting a deletion of two base pairs, designated pin3-3, was isolated. PIN3 expression (data not shown) and immunolocalization (Fig. 4e) studies demonstrated that all three alleles were null.

pin3 mutants display defects in differential growth. Gravitropic and phototropic responses as well as apical hook maintenance are reduced in pin 3 mutants (Fig. $2 \mathrm{~b}-\mathrm{d}$ and Table 1).pin 3 seedlings also display shorter hypocotyls and roots than wild-type seedlings when grown in light (Fig. $2 \mathrm{f}$ and Table 1). However, no such differences were observed in dark-grown seedlings (Fig. 2e and Table 1). Shorter hypocotyl epidermis cells in pin3 suggested that this phenotype was caused by a defect in cell elongation (Fig. $2 \mathrm{~g}, \mathrm{~h}$ and Table 1). The specificity of the pin3 phenotype was further confirmed by identification of two revertants displaying wild-type phenotype among homozygous pin3-2 seedlings. Sequencing of the PIN3 locus in both plants confirmed the excision of the En-1 transposon and restoration of the wild-type PIN3 coding sequence. All defects in pin 3 mutants can be mimicked by growing wild-type plants on auxin efflux inhibitors (Fig. $2 \mathrm{i}$ and Table 1$)^{7,19,20}$, further supporting a role for PIN3 in auxin efflux. The pin3 mutation does not abolish differential growth to the same extent that auxin efflux inhibitors at increased concentrations do. This suggests some level of functional redundancy between PIN3 and other PIN protein(s).

Northern blot analysis revealed the presence of PIN3 transcripts a

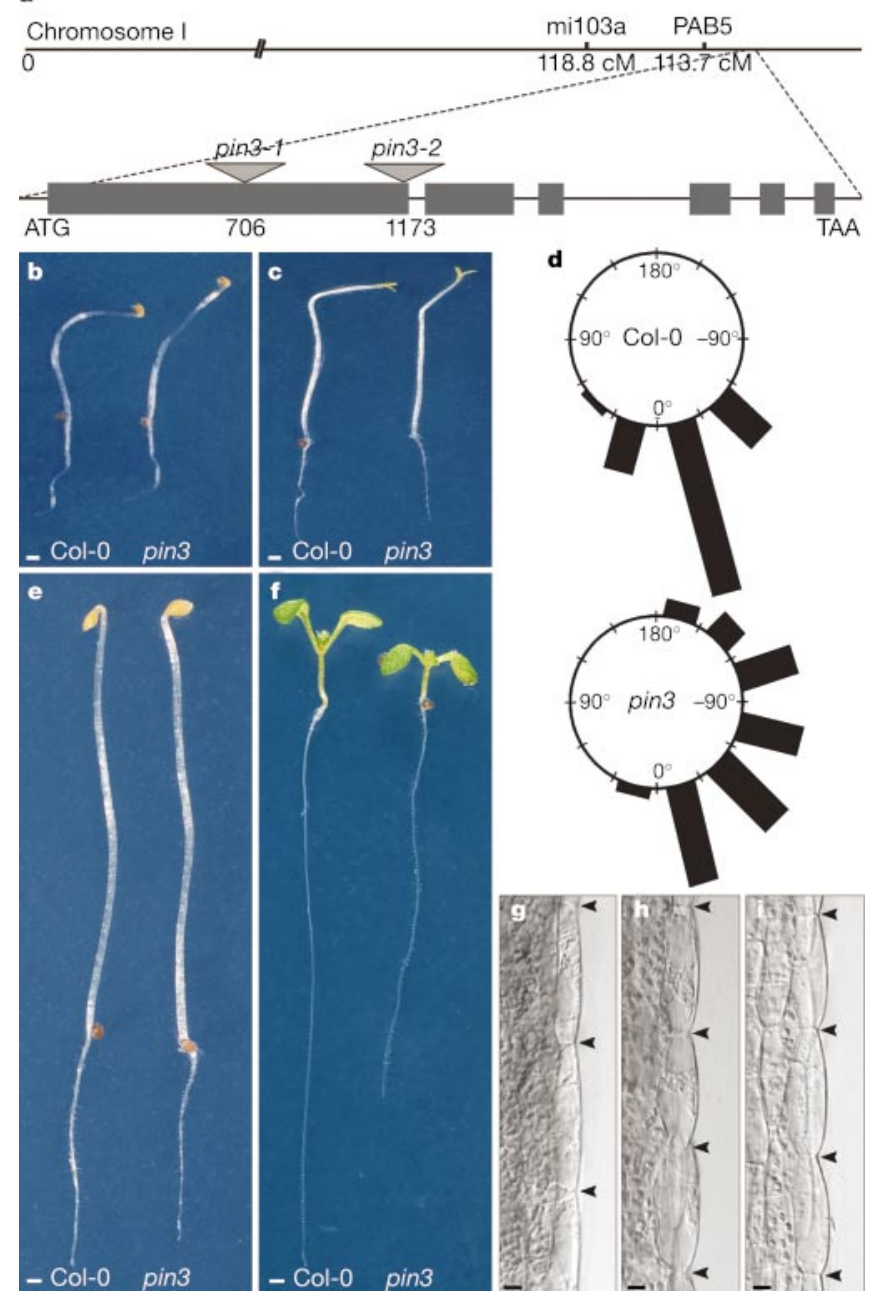

Figure 2 pin3 mutants. a, Exon/intron organization of PIN3 on chromosome 1 showing positions of En-1 elements in pin3-1 and -2 alleles. b, c, pin3 hypocotyls are defective in gravitropic (b) as well as phototropic (c) responses. Scale bars, $500 \mu \mathrm{m}$. d, pin3 mutants are defective in root gravitropism. Each gravistimulated root was assigned to one of twelve $30^{\circ}$ sectors. The length of each bar represents the percentage of seedlings showing direction of root growth within that sector. e, f, Dark-grown pin3 seedlings (e) have the same length of hypocotyls and roots as wild type, but display defects in apical hook maintenance. Light-grown pin3 seedlings (f) display shorter hypocotyls and roots compared with wild type. Scale bars, $500 \mu \mathrm{m}$. g-i, Hypocotyl epidermis cells of pin3 (h) and NPA-grown wild-type (i) seedlings show reduced length compared with untreated wild type $(\mathbf{g})$. Arrowheads indicate cell boundaries. Scale bars, $10 \mu \mathrm{m}$. The following pin3 alleles are depicted: $\operatorname{pin} 3-1(\mathbf{b})$, pin3-2 (c, $\mathbf{d})$ and $\operatorname{pin} 3-3(\mathbf{e}, \mathbf{f}, \mathbf{h})$.

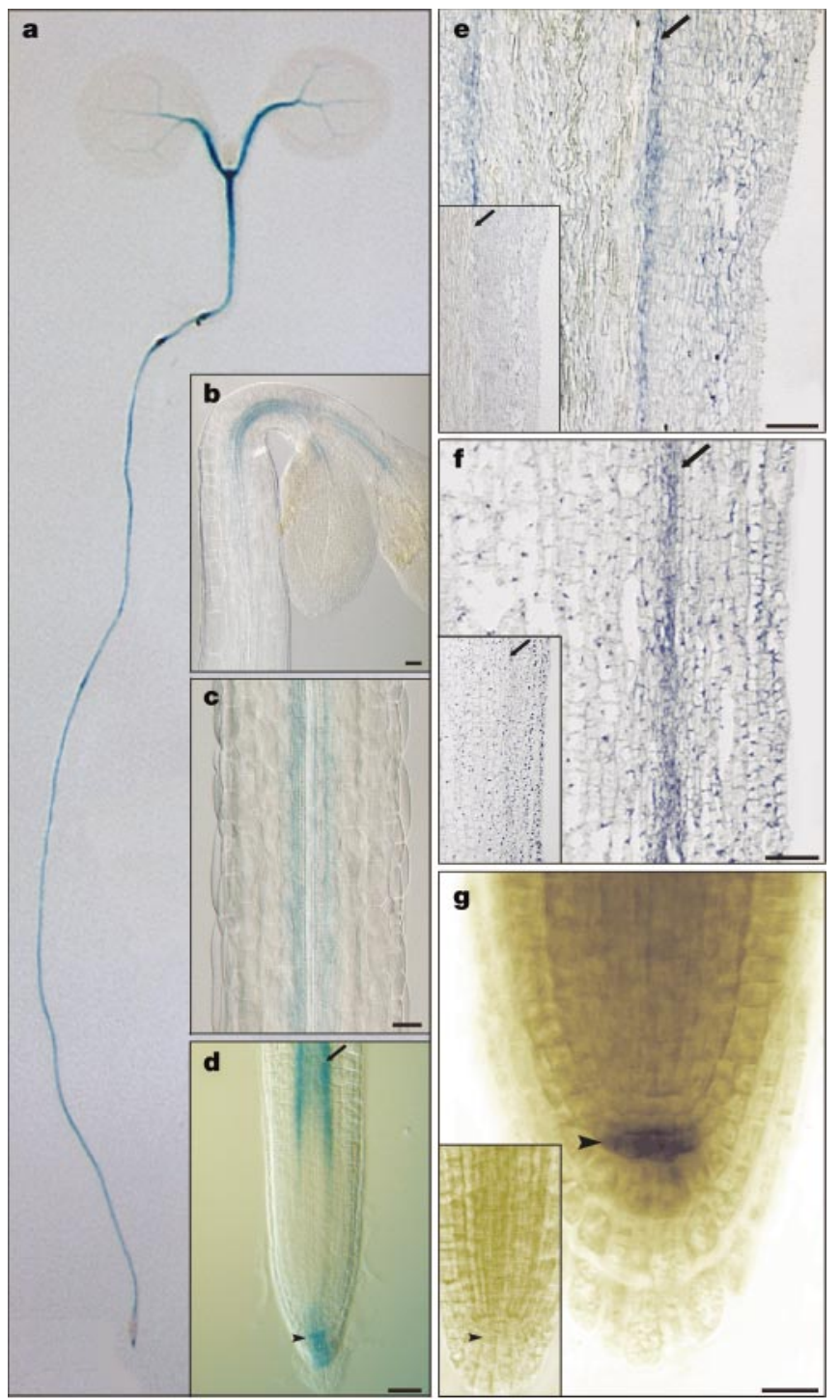

Figure 3 Expression of the PIN3 gene. a-d, PIN3::GUS transgenic seedlings show GUS staining in the apical hook $(\mathbf{b})$, around the hypocotyl vasculature $(\mathbf{c})$, and in the root pericycle (arrow) and columella (arrowhead) (d). Scale bars, $50 \mu \mathrm{m}$. e-g, PIN3 mRNA was detected by in situ hybridization in hypocotyl (e), stem (f) starch sheath (arrows) and root columella (arrowheads) $(\mathbf{g})$ cells. Insets show sense controls $(\mathbf{e}-\mathbf{g})$. Scale bars: $200 \mu \mathrm{m}(\mathbf{e}), 100 \mu \mathrm{m}(\mathbf{f})$ and $20 \mu \mathrm{m}(\mathbf{g})$. Longitudinal sections (e, f) and whole-mount preparations $(\mathbf{a}-\mathbf{d}, \mathbf{g})$ are shown. 


\begin{tabular}{|c|c|c|c|c|c|c|}
\hline Measurement & Col-0 & $(n)$ & pin3 & $(n)$ & $\mathrm{NPA}^{*}$ & $(n)$ \\
\hline Hypocotyl length in light (mm) & $2.21 \pm 0.16$ & (58) & $1.68 \pm 0.15$ & (66) & $1.43 \pm 0.14$ & (56) \\
\hline Root length in light $(\mathrm{cm})$ & $2.41 \pm 0.22$ & (58) & $1.70 \pm 0.16$ & (66) & $1.67 \pm 0.16$ & (56) \\
\hline Hypocotyl length in dark (cm) & $1.32 \pm 0.18$ & (54) & $1.35 \pm 0.12$ & (62) & $1.41 \pm 0.15$ & (62) \\
\hline Root length in dark (cm) & $0.77 \pm 0.07$ & (54) & $0.78 \pm 0.14$ & (62) & $0.85 \pm 0.11$ & (62) \\
\hline Hypocotyl cell length ( $\mu \mathrm{m})$ & $101.8 \pm 10.2$ & (63) & $75.5 \pm 8.40$ & (54) & $72.2 \pm 7.90$ & (61) \\
\hline Hypocotyl gravitropism (deg) & $78.1 \pm 16.9$ & (78) & $31.8 \pm 15.0$ & (81) & - & - \\
\hline Hypocotyl phototropism (deg) & $72.5 \pm 15.5$ & (65) & $40.0 \pm 9.70$ & (72) & - & - \\
\hline Opened apical hooks (\%) & $16.2 \pm 9.67$ & (81) & $65.4 \pm 8.02$ & (97) & - & - \\
\hline
\end{tabular}

Wild-type plants treated with NPA.

predominantly in the shoot and root (data not shown). The staining of transgenic seedlings carrying the PIN3 promoter fused to the $\beta$ glucuronidase (GUS) gene revealed PIN3::GUS expression associated with vasculature (Fig. 3a). Detailed inspection revealed GUS staining in the apical hook of the etiolated seedling (Fig. 3b), in the shoot endodermis (starch sheath) around the vasculature (Fig. 3c), and in the root pericycle and columella (Fig. 3d). To localize the PIN3 protein, PIN3-specific antibodies were raised and immunolocalization experiments performed. The PIN3 protein was found at the periphery of shoot starch sheath as well as root pericycle and columella cells (Fig. $4 \mathrm{a}-\mathrm{e}$ ). Longitudinal sections revealed that PIN3 is localized in a polar way predominantly at the lateral, inner side of the starch sheath as well as root pericycle cells (Fig.
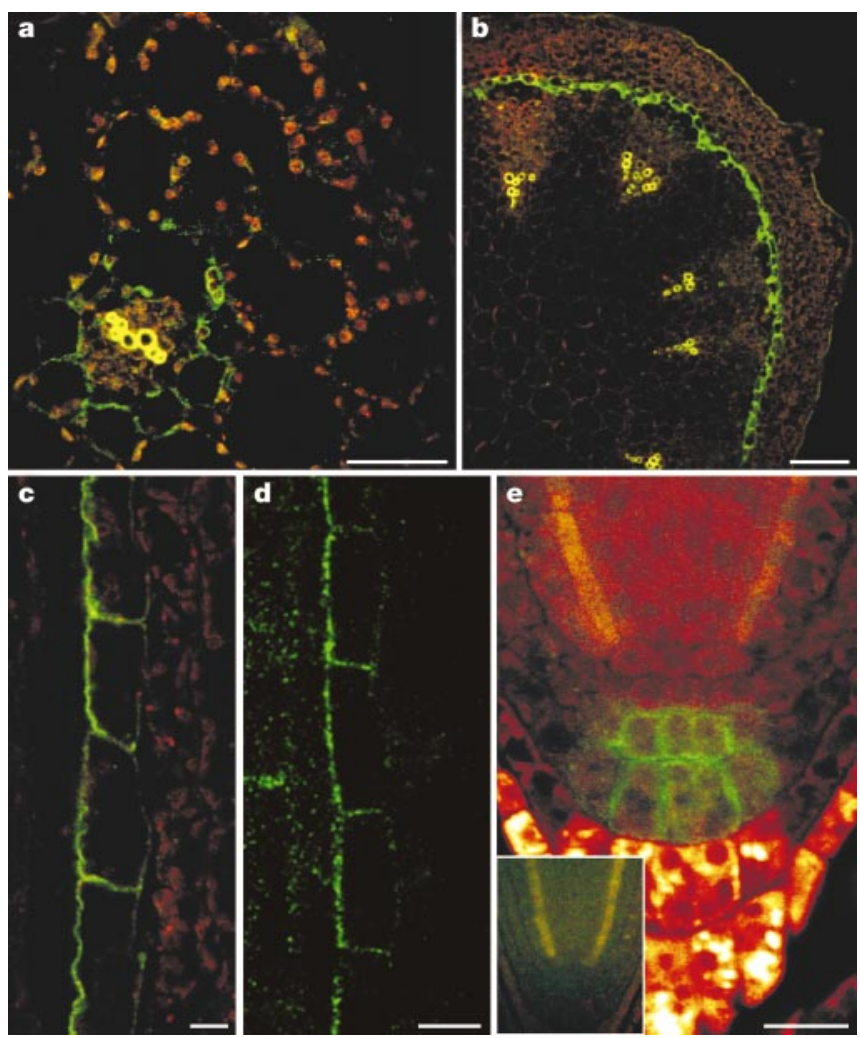

Figure $\mathbf{4}$ Localization of the PIN3 protein. a, b, PIN3 protein localizes to the surface of hypocotyl (a) and stem (b) starch sheath cells. Scale bars, $50 \mu \mathrm{m}$ (a) and $100 \mu \mathrm{m}$ (b). c, d, Longitudinal view of stem starch sheath (c) and root pericycle (d) cells shows lateral Iocalization of PIN3 at the inner cell surface. Scale bars: $10 \mu \mathrm{m}$ (c) and $5 \mu \mathrm{m}$ (d). e, In the root tip, PIN3 is found uniformly in the columella cell boundaries. Inset shows no PIN3 staining in the pin3 mutant. Scale bar, $20 \mu \mathrm{m}$. Transversal (a, b) and longitudinal (c) sections, and whole-mount $(\mathbf{d}, \mathbf{e})$ preparations are shown. The PIN3 protein signal is visualized by green coloration.
$4 c, d)$. In a minor portion of the cells an additional basal or uniform localization of PIN3 was observed (data not shown). In contrast, only uniform distribution of PIN3 in root columella cells was detected (Fig. 4e). No PIN3 signal was detected in pin3 knockout mutants (Fig. 4e, inset). PIN3 messenger RNA localization using in situ hybridization confirms the GUS and immunolocalization staining patterns (Fig. $3 \mathrm{e}-\mathrm{g}$ ).

Anti-PIN3 immunogold labelling revealed signals at the plasma membrane (Fig. 5a) and frequently in vesicles of about $70 \mathrm{~nm}$ diameter, similar in size to exocytotic vesicles (Fig. 5b), suggesting a dynamic subcellular movement of PIN3. The PIN3 protein appears to cycle rapidly between the plasma membrane and undefined endosomal compartments, since the incubation of seedlings with the exocytosis inhibitor Brefeldin A (BFA) led to internalization of PIN3 (Fig. 5d, compare with c), also in the presence of the protein synthesis inhibitor cycloheximide (Fig. 5f, compare with e). Pericycle cells show an accumulation of the PIN3 label in perinuclear compartments (Fig. 5d), very similar to previously described BFA compartments ${ }^{21}$. In contrast, in columella cells PIN3 internalizes in smaller compartments without any regular
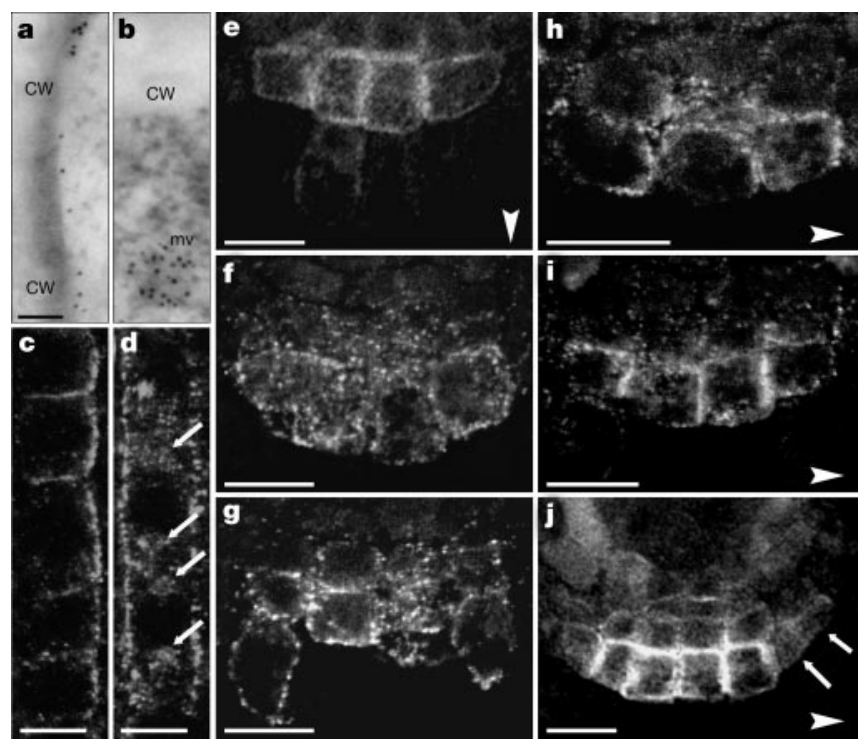

Figure 5 Subcellular localization, actin-dependent cycling and gravity-dependent asymmetric relocation of the PIN3 protein. $\mathbf{a}, \mathbf{b}$, Immunogold electron microscopy shows PIN3 at the plasma membrane (a) and in vesicles (b). cW, cell wall; mv, membrane vesicle. Scale bars, $50 \mathrm{~nm}$. c-g, Actin-dependent cycling of PIN3. Localization of PIN3 in columella $(\mathbf{e}-\mathbf{g})$ and pericycle $(\mathbf{c}, \mathbf{d})$ cells. Internalization of PIN3 after BFA (d), cycloheximide/BFA (f) and latrunculin B (g) treatments. Untreated controls (c, e) are shown. Perinuclear compartments are indicated by arrows in $\mathbf{d}$. Scale bars, $5 \mu \mathrm{m}(\mathbf{c}, \mathbf{d})$ and $10 \mu \mathrm{m}(\mathbf{e}-\mathbf{g}) \cdot \mathbf{h}-\mathbf{j}$, Relocation of PIN3 in columella cells after a change in the gravity vector after $2 \mathrm{~min}$ (h) and $10 \mathrm{~min}$ (i). After $1 \mathrm{~h}$ the PIN3 localization domain expands in lateral root cap (indicated by arrows) and in columella initials (j). Scale bars, $10 \mu \mathrm{m}$. The apparent gravity vectors are indicated by arrowheads 
positioning (Fig. 5f). A similar pattern of PIN3 localization was observed when the actin cytoskeleton was disrupted by latrunculin B (Fig. 5g) or cytochalasin D (data not shown) treatment, suggesting an actin-dependent cycling of PIN3. A similar cycling pattern has been demonstrated for the homologous PIN1 protein; however, the biological meaning of this process remains unclear ${ }^{21}$. PIN3, in contrast to PIN1, was frequently detected in vesicles, suggesting that PIN3 cycles more rapidly or that the equilibrium of intracellular PIN3 pools is shifted more in favour of the internalized PIN3 pool.

The rapid PIN3 cycling in gravity-sensing cells (starch sheath, columella) ${ }^{22}$ raised the possibility that this cycling may provide the mechanism for rapid relocalization of PIN3 after perception of an environmental stimulus. We examined the localization of the PIN3 protein in columella cells after a change in the gravity vector. Already at $2 \mathrm{~min}$ after root rearrangement, the originally symmetric PIN3 distribution (Fig. 5e) becomes asymmetric, with most of the PIN3 protein at the lateral side of columella cells (Fig. 5h). After 5 min the relocalization of PIN3 protein appeared to be complete (Fig. 5i), and was maintained up to $20 \mathrm{~min}$ afterwards (data not shown). One hour after gravity stimulation the asymmetry of the PIN3 localization was observed at the tissue level. PIN3 protein was detected in an enlarged, asymmetric localization domain including columella initial and lateral root cap cells (Fig. 5j, compare with e and Fig. 4e).

Our findings provide the molecular evidence in support of the classical Cholodny-Went model for tropic growth ${ }^{2}$. We show that asymmetric growth correlates with auxin efflux-dependent asymmetric auxin distribution and requires laterally localized PIN3 protein. Developmental requirements for PIN3, its localization, as well as the proposed biochemical function for PIN proteins make PIN3 a likely candidate for an efflux component of the lateral auxin transport system. The actin-dependent cycling and rapid relocalization of PIN3 in cells containing gravity-sensing statoliths ${ }^{22}$, taken together with previous findings supporting a direct role of the actin cytoskeleton in gravity perception ${ }^{23,24}$, imply a cellular mechanism for the regulation of asymmetric auxin distribution and differential growth. After a change of the gravity vector, actin-enmeshed statoliths ${ }^{25}$ sediment, causing reorganization of the actin cytoskeleton. Thus PIN3 is redirected towards one side of the columella cells and determines direction of auxin flux, which leads to asymmetric auxin accumulation and differential growth. It is possible that tropic responses of shoots are regulated by a similar mechanism, as the loss of PIN3 or the PIN3-containing starch sheath correlate with the shoot agravitropic phenotype ${ }^{26}$.

\section{Methods}

\section{Materials used}

The PIN3 gene was identified in a bacterial artificial chromosome genomic library (http:// www.mpimp-golm.mpg.de/mpi-mp-map/) with PIN1 probe (nucleotides 1-385; GenBank accession number AF089084). The full-length PIN3 cDNA was isolated from a stem cDNA library (GenBank accession number AF087818). The PIN3::GUS construct was generated by fusion of a PCR-amplified fragment (nucleotides -1,764 to -1) upstream of the ATG codon and the GUS gene. We used the following probes and primers: nucleotides 999-1449 of the PIN3 cDNA, 5' -TCCTCTCACTTCTTCTTCTTCCTC-3'; $5^{\prime}$-TTTATTT ATCTTTTCTTTGTCTCG-3'.

\section{Phenotype analyses}

Seedlings were grown as described previously ${ }^{10}$. For hypocotyl bending, 3-day-old etiolated DR5::GUS, Col-0 (Columbia ecotype) and pin3 seedlings were transferred and orientated on vertical plates with or without $10 \mu \mathrm{M} 1-\mathrm{N}$-naphthylphthalmic acid (NPA), immediately subjected to the gravity or unilateral light stimulation for $20 \mathrm{~h}$ stained for GUS activity, and photographed. The root gravitropism was scored in the dark in 3-dayold, light-grown seedlings, $6 \mathrm{~h}$ after turning the roots $135^{\circ}$. The quantification of responses was performed with Adobe Illustrator software. Apical hook opening was scored $50 \mathrm{~h}$ after germination in vertically grown, etiolated seedlings. The described defects were observed in all alleles examined (pin3-1, -2 and -3).

\section{Expression and localization analysis}

Histochemical staining for GUS activity, northern blot analysis, in situ hybridization, immunolocalization and immunogold electron microscopy were performed as described $^{10,12}$. PIN3-specific antibodies were generated using a recombinant protein corresponding to amino acids 334-483. Affinity-purified primary anti-PIN3, fluorescein isothiocyanate-conjugated and CY3-conjugated anti-rabbit secondary antibodies (Dianova) were diluted 1:40, 1:200 and 1:600, respectively. Brefeldin A (Molecular Probes) and latrunculin B (Duchefa) treatments were performed with corresponding controls before immunolocalization as described ${ }^{21}$. For the gravitropism treatment, before immunolocalizations, plates with vertically grown seedlings were placed horizontally, and samples were fixed directly on plates at specific time points.

Received 17 October; accepted 10 December 2001.

1. Darwin, C. \& Darwin, F. in Darwins Gesammelte Werke Bd. 13 (Schweizer-bart'sche Verlagsbuchhandlung, Stuttgart, 1881).

2. Went, F. W. Reflections and speculations. Annu. Rev. Plant Physiol. 25, 1-26 (1974).

3. Hart, J. W. Plant Tropism and Other Movements (Unwin Hyman, London, 1990).

4. Epel, B. L., Warmbrodt, R. P. \& Bandurski, R. S. Studies on the longitudinal and lateral transport of IAA in the shoots of etiolated corn seedlings. J. Plant Physiol. 140, 310-318 (1992).

5. Li, Y., Hagen, G. \& Guilfoyle, T. J. An auxin-responsive promoter is differentially induced by auxin gradients during tropisms. Plant Cell 3, 116-1176 (1991).

6. Luschnig, C., Gaxiola, R. A., Grisafi, P. \& Fink, G. R. EIR1, a root specific protein involved in auxin transport, is required for gravitropism in Arabidopsis thaliana. Genes Dev. 12, 2175-2187 (1998).

7. Friml, J. \& Palme, K. Polar auxin transport-old questions and new concepts. Plant Mol. Biol. (in the press).

8. Palme, K. \& Gälweiler, L. PIN-pointing the molecular basis of auxin transport. Curr. Opin. Plant Biol. 2, 375-381 (1999).

9. Rubery, P. H. \& Sheldrake, A. R. Carrier-mediated auxin transport. Planta 118, 101-121 (1974).

10. Müller, A. et al. AtPIN2 defines a locus of Arabidopsis for root gravitropism control. EMBO J. 17, 6903-6911 (1998).

11. Rashotte, A. et al. Basipetal auxin transport is required for gravitropism in roots of Arabidopsis. Plant Physiol. 122, 481-490 (2000).

12. Gälweiler, L. et al. Regulation of polar auxin transport by AtPIN1 in Arabidopsis vascular tissue. Science 282, 2226-2230 (1998).

13. Morris, D. A. \& Thomas, A. A microautoradiographic study of auxin transport in the stem of intact pea seedlings (Pisum sativum L.) J. Exp. Bot. 29, 147-157 (1978).

14. Biorkman, T. \& Cleland, R. E. The role of the epidermis and cortex in gravitropic curvature of maize roots. Planta 176, 513-518 (1988).

15. Ulmasov, T., Murfett, J., Hagen, G. \& Guilfoyle, T. J. Aux/1AA proteins repress expression of reporter genes containing natural and highly active synthetic auxin response elements. Plant Cell 9, 1963-1971 (1997)

16. Casimiro, I. et al. Auxin transport promotes Arabidopsis lateral root initiation. Plant Cell 13, 843-852 (2001)

17. Friml, J. et al. AtPIN4 mediates sink driven auxin gradients and patterning in Arabidopsis roots. Cell (in the press).

18. Wisman, E., Cardon, G. H., Fransz, P. \& Saedler, H. The behaviour of the autonomous maize transposable element En/Spm in Arabidopsis thaliana allows efficient mutagenesis. Plant Mol. Biol. 37, 989-999 (1998).

19. Ruegger, M. et al. Reduced naphthylphthalamic acid binding in the tir3 mutant of Arabidopsis is associated with a reduction in polar auxin transport and diverse morphological defects. Plant Cell $\mathbf{9}$, 745-757 (1997).

20. Jensen, P. J., Hangarter, R. P. \& Estelle, M. Auxin transport is required for hypocotyl elongation in light-grown Arabidopsis. Plant Physiol. 16, 455-462 (1998).

21. Geldner, N. et al. Polar auxin transport inhibitors block PIN1 cycling and vesicle trafficking. Nature 413, 425-428 (2001).

22. Hasenstein, K. H. Gravisensing in plants and fungi. Adv. Space Res. 24, 677-685 (1999).

23. Sievers, A., Sondag, C., Trebacz, K. \& Hejnowicz, Z. Gravity induced changes in intracellular potentials in statocytes of cress roots. Planta 197, 392-394 (1995).

24. Monshausen, G. B., Zieschang, H. E. \& Sievers, A. Differential proton secretion in the apical elongation zone caused by gravistimulation is induced by a signal from the root cap. Plant Cell Environ. 19, 1408-1414 (1996).

25. Collings, D. A., Zsuppan, G., Allen, N. S. \& Blancaflor, E. B. Demonstration of prominent actin filaments in the root columella. Planta 212, 392-403 (2001).

26. Fukaki, H. et al. Genetic evidence that the endodermis is essential for shoot gravitropism in Arabidopsis thaliana. Plant J. 14, 425-430 (1998).

\section{Acknowledgements}

We thank G. Jürgens for enabling J.F. to accomplish part of this work in his laboratory; P. Tänzler and M. Sauer for technical assistance; H. Vahlenkamp for technical assistance in immunocytochemistry; M. Estelle for providing material and suggestions; T. Altman for BAC filter sets; the ADIS (Automated DNA Isolation and Sequencing) service group for DNA sequencing; ZIGIA (Center for Functional Genomics in Arabidopsis) for the En lines; and N. Geldner, T. Hamann, G. Jürgens, K. Schrick and C. Schwechheimer for comments and critical reading of the manuscript. This work was supported by a fellowship of the DAAD (J.F.), the DFG (Schwerpunktprogramm Phytohormone), the Fonds der chemischen Industrie, the European Communities Biotechnology Programs, the INCOCopernicus Program and the European Space Agency MAP-Biotechnology Programme.

\section{Competing interests statement}

The authors declare that they have no competing financial interests.

Correspondence and requests for materials should be addressed to J.F. (e-mail: jiri.friml@zmbp.uni-tuebingen.de) or K.P. (e-mail: palme@mpiz-koeln.mpg.de). 